\title{
miR-361-3p regulates FSH by targeting FSHB in a porcine anterior pituitary cell model
}

\author{
Rui-Song Ye*, Meng Li*, Chao-Yun Li, Qi-En Qi, Ting Chen, Xiao Cheng, Song-Bo Wang, \\ Gang Shu, Li-Na Wang, Xiao-Tong Zhu, Qing-Yan Jiang, Qian-Yun Xi and \\ Yong-Liang Zhang \\ Chinese National Engineering Research Center for Breeding Swine Industry, SCAU-Alltech Research Joint Alliance, \\ Guandong Provincial Key Lab of Agro-Animal Genomics and Molecular Breeding, College of Animal Science, \\ South China Agricultural University, Guangzhou, China \\ Correspondence should be addressed to Q-Y Xi or Y-L Zhang; Email: xqy0228@163.com or Zhangyl@scau.edu.cn \\ *(R-S Ye and M Li contributed equally to this work)
}

\begin{abstract}
FSH plays an essential role in processes involved in human reproduction, including spermatogenesis and the ovarian cycle. While the transcriptional regulatory mechanisms underlying its synthesis and secretion have been extensively studied, little is known about its posttranscriptional regulation. A bioinformatics analysis from our group indicated that a microRNA (miRNA; miR-361-3p) could regulate FSH secretion by potentially targeting the FSHB subunit. Herein, we sought to confirm these findings by investigating the miR-361-3p-mediated regulation of FSH production in primary pig anterior pituitary cells. Gonadotropin-releasing hormone (GnRH) treatment resulted in an increase in FSHB synthesis at both the mRNA, protein/hormone level, along with a significant decrease in miR-361-3p and its precursor (pre-miR-361) levels in time- and dose-dependent manner. Using the Dual-Luciferase Assay, we confirmed that miR-361-3p directly targets FSHB. Additionally, overexpression of miR-361-3p using mimics significantly decreased the FSHB production at both the mRNA and protein levels, with a reduction in both protein synthesis and secretion. Conversely, both synthesis and secretion were significantly increased following miR-361-3p blockade. To confirm that miR-361-3p targets FSHB, we designed FSH-targeted siRNAs, and co-transfected anterior pituitary cells with both the siRNA and miR-361-3p inhibitors.

Our results indicated that the siRNA blocked the miR-361-3p inhibitor-mediated upregulation of FSH, while no significant effect on non-target expression. Taken together, our results demonstrate that miR-361-3p negatively regulates FSH synthesis and secretion by targeting FSHB, which provides more functional evidence that a miRNA is involved in the direct regulation of FSH.

Reproduction (2017) 153 341-349
\end{abstract}

\section{Introduction}

The gonadotropin $\mathrm{FSH}$, which is secreted by the anterior pituitary gland, plays an essential role in animal reproduction: it promotes antral follicle development in females and stimulates spermatogenesis in the Sertoli cells in males (Bernard et al. 2010). FSH is a heterodimeric glycoprotein composed of a unique $\beta$ subunit (FSHB) and a common $\alpha$ subunit $(\alpha-\mathrm{GSU})$ that is shared by chorionic gonadotropin (CG), LH and TSH. Many aspects of the transcriptional regulation of $\mathrm{FSH}$ synthesis and secretion have been revealed in recent decades. For example, $\mathrm{FSH}$ is mainly under the control of GnRH and TGF- $\beta$ signaling (Wurmbach et al. 2001, Thackray et al. 2010). Recently, adiponectin was shown to play an important role in FSH secretion during the estrous cycle (Kiezun et al. 2014). However, little is known about the posttranscriptional regulation of FSH production.
microRNAs (miRNAs) are small non-coding RNAs (-22 nt) that regulate gene expression at the posttranscription level by suppressing translation or degrading their target mRNA (Ambros 2004, Karginov et al. 2010). The role of miRNAs in pituitary hormone regulation is increasingly evident, for example, miR-26b was shown to be involved in growth hormone regulation by targeting LEF-1 (Zhang et al. 2010), while miR-375 was found to regulate pituitary pro-opiomelanocortin (POMC) expression (Zhang et al. 2013). To date, several studies have reported either direct or indirect role for miRNAs in the regulation of gonadotropins. Gonadotrope-specific deletion of Dicer, an endoribonuclease responsible for miRNA processing, results in severe suppression of gonadotropin production and fertility defects (Wang et al. 2014). Let-7b/c was found to enhance the stability of the common subunit $\alpha$-GSU indirectly through downregulation of KSRP (Repetto et al. 2012). 
miR-200b/miR-429 was found to promote LH secretion by targeting ZEB1 (Hasuwa et al. 2013), while it was reported that miR-325-3p inhibits LH synthesis and secretion by targeting $L H \beta$ (Nemoto et al. 2012). A recent study showed that miR-132/212 is involved in $\mathrm{GnRH}$-induced FSH expression by targeting SIRT1 and subsequently reducing the FOXO1-mediated inhibition of FSHB transcription (Lannes et al. 2015). In addition, Dang et al. showed that decrease of miR$22-3 p$ is associated with FSH increase in the plasma of Han Chinese patients with premature ovarian failure (Dang et al. 2015). However, it is still unknown whether miRNAs can directly regulate $\mathrm{FSH}$ synthesis or secretion by targeting FSHB. miR-361-3p, an X-linked miRNA, is detected in reproductive organs, including testis (Wu et al. 2014), ovary (Sontakke et al. 2014) and exosome of follicular fluid (Sohel et al. 2013). Additionally, miR-361-3p was frequently differentially expressed in multiple reproduction processes including GnRH induction of FSH secretion (Ye et al. 2013), fetal ovarian development change (Veiga-Lopez et al. 2013) and atretic follicle (Sontakke et al. 2014), which are closely linked to FSH regulation. Most importantly, a bioinformatics study from our group predicted that miR-361-3p may be involved in FSH secretion by targeting FSHB mRNA (Ye et al. 2013). Accordingly, in the present study, we hypothesized that miR-361-3p regulates $\mathrm{FSH}$ by targeting $F S H B$, and examined the relationship between miR-361-3p and FSHB 3'-UTR using the luciferase reporter assay, and investigated intracellular FSHB synthesis and extracellular $\mathrm{FSH}$ secretion following overexpression or knockdown of miR-361-3p in pig anterior pituitary cells. Moreover, the miR-361-3p binding specificity to the target FSHB was further confirmed by co-transfection with miR-361-3p inhibitors and siRNAs.

\section{Materials and methods}

\section{Animals}

The animal experiments were conducted in accordance with the Review of Welfare and Ethics of Laboratory Animals guidelines approved by the Guangdong Province Administration Office of Laboratory Animals, and all experiments were conducted as described in the animal protocol (SCAU-AEC-2010-0416) approved by the Institutional Animal Care and Use Committee of South China Agricultural University.

\section{Primary culture of porcine anterior pituitary cells}

Six healthy 7-day-old male piglets (Landrace) were killed, and the primary anterior pituitary cell culture was conducted as previously described (Barb et al. 1990, Lin et al. 2003). Briefly, pituitary glands were removed under sterile conditions, and the anterior lobe was immediately dissected and washed with Dulbecco Modified Eagle's Medium/Nutrient Mixture F12 (DMEM/F12; Life Technologies) supplemented with
$100 \mathrm{IU} / \mathrm{mL}$ penicillin, $100 \mu \mathrm{g} / \mathrm{mL}$ streptomycin and $2 \mathrm{mg} / \mathrm{mL}$ BSA. The glands were then pooled and minced to produce a suspension in the same medium and centrifuged for $10 \mathrm{~min}$ at $2000 \mathrm{rpm}$. After removal of the supernatant, the pellet containing the tissue fragments was incubated at $37^{\circ} \mathrm{C}$ in DMEM/F12 containing $0.25 \%$ trypsin-EDTA (Life Technologies) and $0.25 \%$ collagenase type $\alpha$ in a flask with constant stirring for $30 \mathrm{~min}$. The enzyme-digested pituitary suspension was then centrifuged at $2000 \mathrm{rpm}$ for $5 \mathrm{~min}$, the supernatant was discarded and the cell pellet was resuspended in DMEM/F12 supplemented with antibiotics and BSA, filtered through a $75-\mu \mathrm{m}$ nylon mesh to remove undigested tissue and cell aggregates, and centrifuged at $2000 \mathrm{rpm}$ for $10 \mathrm{~min}$. The supernatant was discarded and the cell pellet was washed twice in DMEM/F12 supplemented with antibiotics and BSA and suspended at $3 \times 10^{5}$ live cells $/ \mathrm{mL}$ in DMEM/F12 supplemented with antibiotics and 10\% FBS (Life Technologies). Finally, six well plates were seeded with $2 \mathrm{~mL} /$ well of the cell suspension and cultured at $37^{\circ} \mathrm{C}$ in a humidified, $5 \% \mathrm{CO}_{2}$ atmosphere.

\section{GnRH treatment}

When $80-90 \%$ confluent, the pig anterior pituitary cells were serum-starved for $6 \mathrm{~h}$ in DMEM/F12 supplemented with antibiotics and BSA, and then incubated for $12 \mathrm{~h}$ or $24 \mathrm{~h}$ in fresh starvation medium supplemented with $100 \mathrm{nM}$ GnRH (Okada et al. 2003, Yuen et al. 2009, Ye et al. 2013) (Gonadoliberin I; AnaSpec, Fremont, CA, USA). For the dosedependent assay $(10,25,100 \mathrm{nM})$, pituitary cell were treated for $12 \mathrm{~h}$.

\section{Anterior pituitary tissues collection}

Eight anterior pituitary tissues were collected from eight male pigs (13-16 weeks). In brief, pituitary glands were removed and the anterior lobe was immediately dissected from each pituitary gland. Eight anterior pituitary glands were immediately frozen in liquid nitrogen until RNA extraction. Total RNAs were used to quantify FSHB mRNA and miR-361-3p expression by qRTPCR analysis.

\section{FSHB mRNA 3'-UTR plasmid construction}

Based on the miR-361-3p seed sequence, wild-type (WT) or seed-mutated (MT) and seed-deleted (DT) FSHB 3'-UTR sequences, where the binding site (CTGGGG) of miR-361-3p was mutated or deleted, respectively, were chemically synthesized (Sangon, Shanghai, China). The sequences are as follows: WT sense oligo, 5'-CTAGAGGAGGAGCTCCAGGAA TGCAGAGTGCTGGGGCCTCAGTCCTATCACCACTCGTT-3'; antisense, 5'-AACGAGTGGTGATAGGACTGAGGCCCCAGCA СТCTGCATTCСТGGAGСТССТССТ-3'; MT sense oligo, 5'-CTA GAGGAGGAGCTCCAGGAATGCAGAGTGgatatcCCTCAGTC CTATCACCACTCGTT-3'; antisense, 5'-AACGAGTGGTGATAG GACTGAGGGATATCCACTCTGCATTCCTGGAGCTCCTCCT3'; DT sense oligo, 5'-CTAGAGGAGGAGCTCCAGGAATGC AGAGTGCCTCAGTCCTATCACCACTCGTT-3'; and antisense, 5'-AACGAGTGGTGATAGGACTGAGGCACTCTGCATTCCT GGAGCTCCTCCT-3'. The complementary oligonucleotides 
were resuspended at a $1: 1$ ratio $(1 \mu \mathrm{g} / \mu \mathrm{L}$ each) in an annealing buffer (10 mM Tris, $\mathrm{pH} 7.5-8.0,50 \mathrm{mM} \mathrm{NaCl}$ and $1 \mathrm{mM}$ EDTA) and heated to $95^{\circ} \mathrm{C}$ for $10 \mathrm{~min}$ to remove secondary structures. The temperature was then gradually reduced until room temperature was reached. The annealed products were then cloned into the pGL3 control vector (Promega) downstream of the luciferase gene.

\section{Luciferase reporter assay}

$\mathrm{CHO}$ cells were seeded in 24-well cell culture plates $\left(1.5 \times 10^{5}\right.$ cells per well), and cultured in RPMI-1640 (Life Technologies) supplemented with $10 \%$ FBS. The next day, the cells were transfected with $50 \mathrm{ng}$ pRL-TK (Renilla luciferase control for normalization; Promega) and 500 ng recombinant luciferase FSHB 3'-UTR reporters, as well as miR-361-3p mimics or a negative control (75 pM, GenePharma, Shanghai, China) using Lipofectamine 2000 (Life Technologies). Cells were harvested $48 \mathrm{~h}$ after transfection, and luciferase activity was determined using a Dual-Luciferase Reporter Assay system, according to the manufacturer's recommendations (Promega).

\section{Alteration of miR-361-3p expression in the porcine anterior pituitary cells}

The porcine pituitary cells were cultured in 12-well plates as described above (see Primary culture of porcine anterior pituitary cells, 12 -well plates were seeded with $2 \times 10^{5}$ live cells/well). When $80-90 \%$ confluent, cells were transfected with $100 \mathrm{nmol} /$ well of a synthetic RNA duplex (miRNA mimics; GenePharma) or a negative control (NC) to upregulate sscmiR-361-3p expression. The sequences are as follows: mimics, 5'-CCCCCAGGUGUGAUUCUGAUUUGC-3' (sense), 5'-AAAUCAGAAUCACACCUGGGGGUU-3' (antisense); NC, 5'-UUCUCCGAACGUGUCACGUTT-3' (sense) and 5'-ACGUGACACGUUCGGGAATT-3' (antisense). Additionally, miR-361-3p inhibition was also achieved by transfecting cells with $100 \mathrm{nmol} /$ well of $2^{\prime}-O$ methylated single-stranded miR-361-3p antisense oligonucleotides (GenePharma). The sequences were as follows: ssc-miR-361-3p inhibitor,
5'-GCAAAUCAGAAUCACACUGGGGG-3'; negative control (i-NC), 5'-CAGUACUUUUGUGUAGUACAA-3'. At $24 \mathrm{~h}$ post-transfection, the supernatants were collected for FSH quantification by radioimmunoassay (RIA), while the cells were subjected to quantitative analysis to examine mRNA and miRNA expression, as well as Western blotting.

\section{Radioimmunoassay}

The supernatants of anterior pituitary cells were collected for FSH quantification by RIA using a double-antibody ${ }^{125} \mathrm{I}-\mathrm{FSH}$ RIA detection kit (Nine Tripods Medical \& Bioengineering Co Ltd., Tianjin, China). The intra- and interassay CVs were $5.5 \%$ and $8.7 \%$, respectively. The minimum assay sensitivity was $1.0 \mathrm{mIU} / \mathrm{mL}$, the primary data from RIA was further calibrated by total protein amount. At least six samples were used for each group.

\section{RNA extraction and $q R T-P C R$ analysis}

Total RNA was extracted from cells using the TRIzol reagent (Life Technologies) following the manufacturer's instructions. Total RNA samples were treated with recombinant DNase I (Ambion) to remove genomic DNA contamination. A minusreverse transcriptase ('-RT') control was set to confirm genomic DNA was completely removed. To assay for either the miRNAs or mRNA, DNase I - treated total RNA $(1 \mu \mathrm{g})$ was subjected to the reverse transcription reaction using $M-M L V$ reverse transcriptase (Promega). Reverse transcription of pre-miR-361 was achieved by gene-specific primers. For miR-361-3p, reverse transcription was achieved using a stem-loop primer; for mRNAs, the first-strand cDNA was synthesized using oligo $(\mathrm{dT})_{18}$ primers, and the cDNA was diluted 5-fold with $\mathrm{ddH}_{2} \mathrm{O}$. A final $20-\mu \mathrm{L}$ volume $q \mathrm{RT}-\mathrm{PCR}$ was performed using the STRATAGENE Mx3005P sequence detection system. The PCR mix contained $2 \mu \mathrm{L}$ cDNA, $10 \mu \mathrm{L} 2 \times$ SYBR Green PCR Master Mix (Toyobo, Osaka, Japan) and $10 \mu \mathrm{M}$ of each primer. The reaction cycle was as follows: $1 \mathrm{~min}$ at $95^{\circ} \mathrm{C}, 40$ cycles of $15 \mathrm{~s}$ at $94^{\circ} \mathrm{C}$ and $15 \mathrm{~s}$ at the corresponding annealing temperature (Tm), and $72^{\circ} \mathrm{C}$ for $40 \mathrm{~s}$, followed by a quick denaturation

Table 1 Primers used for reverse transcription (RT) and qRT-PCR.

\begin{tabular}{|c|c|c|c|c|}
\hline Primer name & Primer sequence $(5 \rightarrow 3)$ & Gene accession & Product length $(\mathrm{bp})$ & $\operatorname{Tm}\left({ }^{\circ} \mathrm{C}\right)$ \\
\hline Pre-miR-361 RT primer & GAGGAGGAGGAAGCAAATCAGA & - & - & - \\
\hline Pre-miR-361-F & AGAATCTCCAGGGGTAC & MI0013145 & 65 & 56 \\
\hline Pre-miR-361-R & GGAGGAAGCAAATCAGA & & & \\
\hline ssc-miR-361-3pRT primer & $\begin{array}{l}\text { GTCGTATCCAGTGCGTGTCGTG } \\
\text { GAGTCGGCAATTGCACTGGATAC } \\
\text { GACGCAAATC }\end{array}$ & - & - & - \\
\hline miR-361-3p-F & CCCCCAGGTGTGATTC & MIMAT0013934 & 55 & 58 \\
\hline miR-361-3p-R & ATCCAGTGCGTGTCGTGGA & & & \\
\hline U6-F & CTCGCTTCGGCAGCACA & NR_004394 & 71 & 58 \\
\hline U6-R & AACGCTTCACGAATTTGCGT & & & \\
\hline FSHB-F & ССАТСТСССААТСТGТСТС & NM_213875.1 & 177 & 58 \\
\hline FSHB-R & GCATTTAGTCCTTTCACCC & & & \\
\hline FOXL2-F & GAGAAGAGGCTCACGCTGTCCG & NM_001244665 & 112 & 58 \\
\hline FOXL2-R & TGAGGCTGAGGTTGTGGCGAAT & & & \\
\hline LHB-F & AGAGCTGAGCTTTGCСТCСАTC & NM_214080.1 & 156 & 60 \\
\hline LHB-R & CGGTCACAGGCCAAGGGTT & & & \\
\hline$\beta$-actin- $\mathrm{F}$ & CCAGCACGATGAAGATCAAGATC & AY550069.1 & 55 & 58 \\
\hline$\beta$-actin-R & ACATCTGCTGGAAGGTGGACA & & & \\
\hline
\end{tabular}


at $95^{\circ} \mathrm{C}$ for $5 \mathrm{~min}, \mathrm{Tm}$, plus a slow ramp from $\mathrm{Tm}$ to $95^{\circ} \mathrm{C}$ to generate a melt curve to control the specificity of the amplified product. The no template control was set as the NC for both the miRNA or mRNA assays. U6 small nuclear RNA and $\beta$-actin were used for internal controls for miR-361-3p and $F S H B$, respectively. The optimization of primers was confirmed by standard curve construction (efficiency is $90 \%-105 \%$, and $\left.R^{2}>0.980\right)$. Table 1 contains the information on all primers used for reverse transcription and qRT-PCR. The $2^{-\Delta C t}$ method was employed to quantify and normalize the expression data.

\section{Western blotting analysis}

Proteins were extracted from the TRIzol-treated samples, as previously described (Chomczynski 1993). In brief, after removing the upper aqueous phase and interphase from Trizollysed supernatant, the lower organic phase was collected and precipitated using isopropanol at room temperature for $10 \mathrm{~min}$. The samples were then centrifuged at $4{ }^{\circ} \mathrm{C}, 12,000 \times \mathbf{g}$ for $10 \mathrm{~min}$ and rinsed three times with $0.3 \mathrm{M}$ guanidine hydrochloride. Next, absolute alcohol was added and the samples were centrifuged at $4{ }^{\circ} \mathrm{C}, 7500 \times \mathrm{g}$ for $5 \mathrm{~min}$. After drying, the proteins were solubilized in 1\% SDS containing $1 \mathrm{mM}$ phenylmethyl sulfonylfluoride, and the total soluble protein was quantified using a BCA protein assay. Protein aliquots $(30 \mu \mathrm{g})$ were separated on a $10 \%$ SDS-PAGE gel, electroblotted onto a polyvinylidene difluoride membrane and blotted with an anti-FSHB antibody (1:1000 dilution, Abcam). $\beta$-Actin (1:2000 dilution, Abcam) was used to normalize protein expression. Images were acquired using FluorChem M (ProteinSimple, Santa Clara, CA, USA), and analyzed using ImageJ software (NIH).

\section{siRNA design and transfection}

The siRNA oligos potentially targeting FSHB, siRNA1 (5'-CCAUGCAGACUCCCUGUAU-3), as well as the corresponding NCs were designed and synthesized by GenePharma. Primary anterior pituitary cells were transfected with either the siRNAs or NCs to select the optimal siRNA concentration. Finally, the siRNAs were co-transfected with the miR-361-3p inhibitor, and FSH levels in the culture supernatants were determined.

\section{Statistical analysis}

Data were presented as mean \pm S.E.M., statistical analysis was performed by SPSS 17.0 software. Statistical significance was determined using the Student's t-test, whereas multiple comparisons were performed by analysis of variance and differences where $P<0.05$ were considered significant. Pearson's correlation coefficient $R$ was used to measure the product-moment coefficient of correlation between miR361-3p and FSHB mRNA abundance.

\section{Results}

\section{Inverse relation between miR-361-3p expression and FSH synthesis}

Previous findings from our group (Ye et al. 2013) indicated that FSH secretion was accompanied by a decrease in miR-361-3p levels. In this study, we directly tested if miR-361-3p modulated $\mathrm{GnRH}$-induced $\mathrm{FSH}$ synthesis. Firstly, to test the dose-dependent effect of
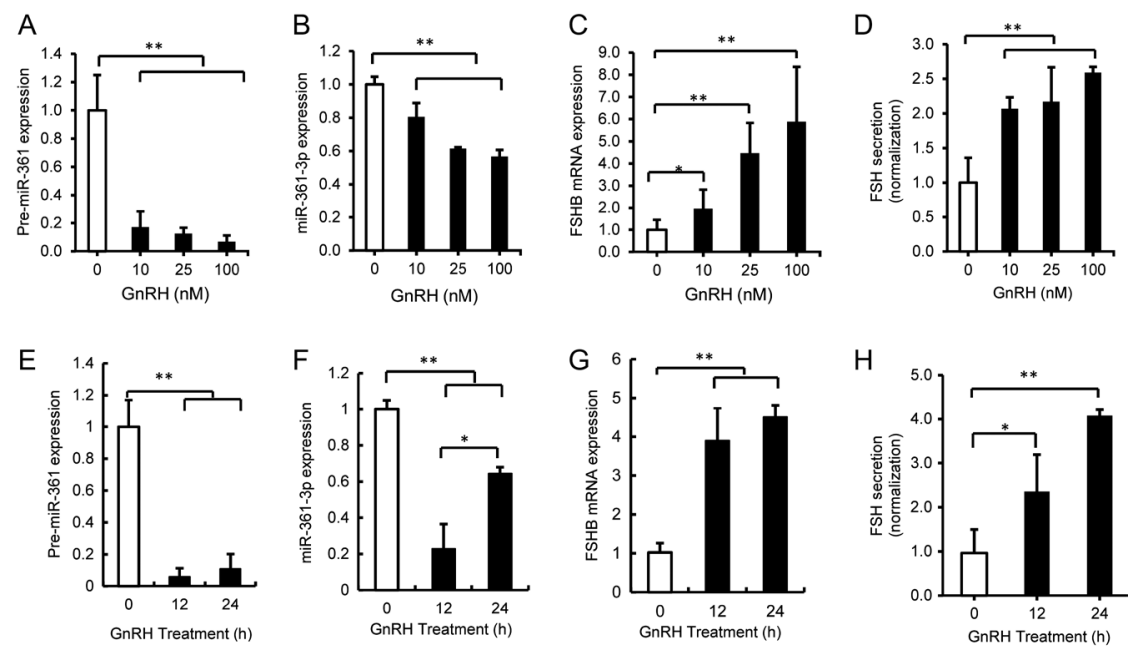

।

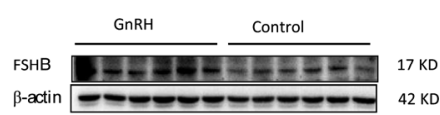

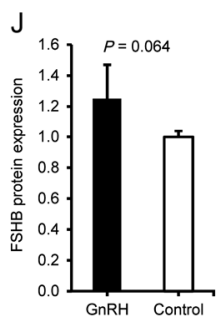

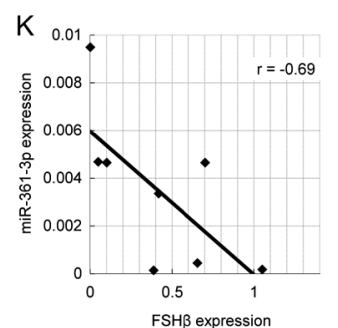

Figure 1 Inverse relation between miR-361-3p expression and FSH synthesis. (A) Primary pig anterior pituitary cells were treated with 10 , 25 and $100 \mathrm{nM} \mathrm{GnRH}$ for $12 \mathrm{~h}$ and subjected to qRT-PCR to examine miR-361-3p precursor (pre-miR-361) expression. (B) Examination of miR-361-3p expression by qRT-PCR. (C) Examination of the FSHB mRNA levels by qRT-PCR. (D) Examination of the FSH secretion in cell supernatant by RIA. (E) Primary pig anterior pituitary cells were treated with $100 \mathrm{nM} \mathrm{GnRH}$ for 12 and $24 \mathrm{~h}$ and subjected to qRT-PCR to examine pre-miR-361 expression. (F) Examination of miR-361-3p expression by qRT-PCR. (G) Examination of the FSHB mRNA levels by qRT-PCR. (H) Examination of the FSH secretion in cell supernatant by RIA. (I) FSHB protein expression after $24 \mathrm{~h} \mathrm{GnRH}$ treatment by Western blotting. (J) Comparison of the relative expression of FSHB following densitometric analysis of blots shown in (I), densitometric analysis was conducted by ImageJ software. (K) Correlation analysis between miR-361-3p and FSHB mRNA abundance in pig anterior pituitary tissues by qRT-PCR. ${ }^{*} P<0.05 ;{ }^{*} P<0.01$; ns, not significant. 
GnRH on miR-361-3p expression, pig anterior pituitary cells were treated with 10,25 and $100 \mathrm{nM} \mathrm{GnRH}$, respectively, and then evaluated FSH expression as well as miR-361-3p abundance at $12 \mathrm{~h}$ after $\mathrm{GnRH}$ treatment. We observed that $\mathrm{GnRH}$ dose-dependently decreased miR-361-3p precursor pre-miR-361, $(83 \%, 88 \%, 95 \%$ decrease, respectively, $P<0.01$, Fig. $1 \mathrm{~A})$ and mature miR-361-3p expression $(21 \%, 40 \%, 42 \%$ decrease, respectively, $P<0.01$, Fig. 1B), but increased $F S H B$ expression (1.95-fold $P<0.05$; 4.46-fold, $P<0.01$; 5.87fold, $P<0.01$, respectively, Fig. $1 \mathrm{C})$ and FSH hormone secretion (2.06-fold, 2.16-fold, 2.59-fold, respectively, Fig. 1D). Furthermore, we test miR-361-3p expression and FSHB expression with GnRH exposure time. Pig anterior pituitary cells were treated with $100 \mathrm{nM} \mathrm{GnRH}$, miR-361-3p and FSH expression were measured at 12 and $24 \mathrm{~h}$ after treatment. The results indicated that both 12 and $24 \mathrm{~h}$ treatment led to an obvious decrease in premiR-361 (95\%, 90\% decrease, respectively, $P<0.01$, Fig. 1E) and mature miR-361-3p (78\%, 37\% decrease, respectively, $P<0.01$, Fig. 1F). It also led to significant increase in FSHB mRNA expression (3.90-fold, 4.80fold over the control group, respectively, $P<0.01$, Fig. 1G) and FSH secretion (2.40-fold, 4.10-fold as the control group, Fig. $1 \mathrm{H}$ ) and an increase tendency of FSHB protein (25\% increase, $P=0.064$, Fig. $1 \mathrm{I}$ and $\mathrm{J})$. Interestingly, similar to the previous study (Yuen et al. 2009, Lannes et al. 2016), we also observed long GnRH exposure time $(24 \mathrm{~h})$ tended to restore pre-miR-361 and miR-361-3p expression. Regardless, these data supported that the GnRH-mediated downregulation of miR-361-3p was involved in FSH synthesis in vitro.

To further test if miR-361-3p has a potential role in FSH synthesis in vivo, a correlation analysis between miR-361-3p level and FSHB mRNA abundance was performed, the result showed miR-361-3p were negatively correlated with FSH mRNA abundance $(r=-0.69, P=0.029)$ (Fig. $1 \mathrm{~K}$ ).

\section{miR-361-3p targets the FSHB 3'-UTR}

We previously reported that $F S H B$ has one putative binding site for miR-361-3p in the 3'-UTR (Ye et al. 2013), and RNAhybrid prediction showed miR-361-3p targets the FSHB 3'-UTR by perfectly binding this site via the seed sequence (a 2-7 nt sequence at the miRNA 5 '-end, CCCCAG) and the adjacent sequences (Fig. 2A). Hence, we hypothesized miR-361-3p regulates FSH production by directly targeting FSHB mRNA. To prove this, the 3'-UTR sequence of $F S H B$ harboring the predicted binding sites for miR-361-3p was inserted downstream of the luciferase gene in pGL3-control luciferase reporter vectors. FSHB 3'-UTR sequences with a mutation or deletion in the seed sequence were also designed and inserted. The constructs were named WT, MT and DT, respectively (Fig. 2B and C). CHO cells were co-transfected with the pGL3-control plasmid as well as the pRL-TK plasmid for normalization, along with miR-361-3p mimics or a NC. Measurement of the relative luciferase activity $48 \mathrm{~h}$ after transfection revealed that transfection with the miR-361-3p mimics reduced the luciferase activity of the WT plasmid significantly $(P<0.01)$, but did not affect that of the MT and DT-FSHB mRNA 3'-UTR plasmids (Fig. 2D). These results indicate that FSHB is a direct target of miR-361-3p.

\section{miR-361-3p overexpression inhibits FSH synthesis and secretion in pituitary cells}

To obtain further evidence that miR-361-3p regulates $\mathrm{FSH}$, we transfected anterior pituitary cells with miR361-3p mimics, which led to a remarkable increase in
A

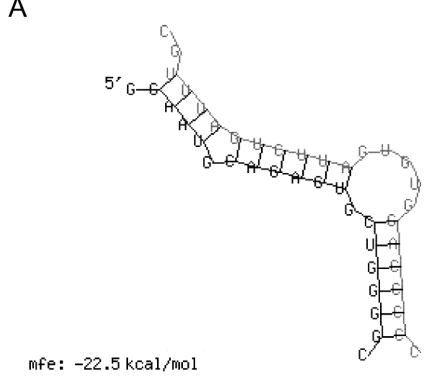

B

Seed sequence

$3^{\prime}$ CGUUUAGUCUUAGUgugGaCCCCC 5' ssc-miRNA-361-3p | ||||

$5^{\prime}$ UCCAGGAAUGCAGAGUGCUGGGGC $3^{\prime}$ pig FSH $\beta 3^{\prime}$-UTR (WT)

$5^{\prime}$ UCCAGGAAUGCAGAGUGGATATCC $3^{\prime}$ pig FSH $33^{\prime}$-UTR (MT)

$5^{\prime}$ UCCAGGAAUGCAGAGUG----a--C $3^{\prime}$ pig FSH $33^{\prime}$-UTR (DT)
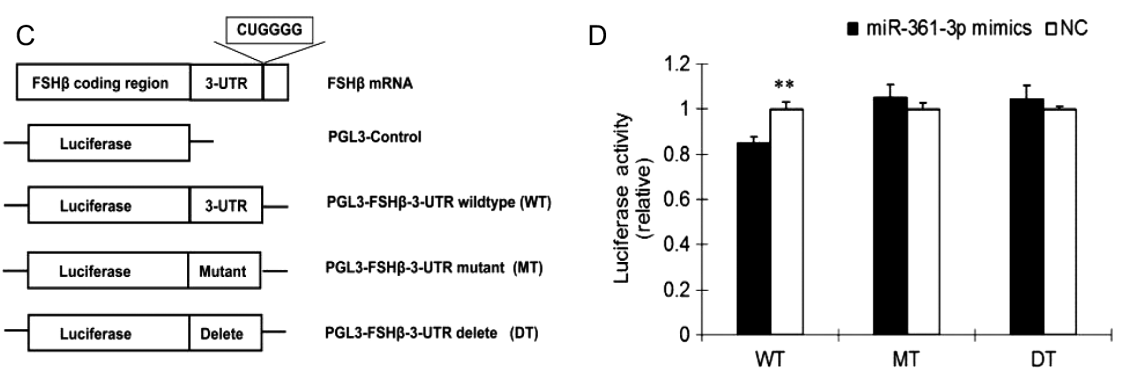

Figure 2 miR-361-3p directly targets FSHB. (A) Predicted secondary structure of miR361-3p for binding to the FSHB 3'-UTR, generated using RNAhybrid. (B) Partial FSHB 3'-UTR sequence in wild-type (WT), mutant (MT) and deleted (DT) constructs. The miR-361-3p seed sequence binding sites were replaced or deleted in MT and DT, respectively. Ssc-miR-361-3p represents the pig miR-361-3p sequence. (C) Schematic of WT, mutant or deleted recombinant luciferase reporter plasmids. WT, MT or DT FSHB mRNA 3'-UTR sequences were cloned downstream of the luciferase reporter gene. (D) DualLuciferase Assay for $\mathrm{CHO}$ cells transfected with reporter plasmids containing FSHB mRNA 3'-UTR WT, MT or DT sequences. ${ }^{*} P<0.05$; ${ }^{*} P<0.01$. 
A
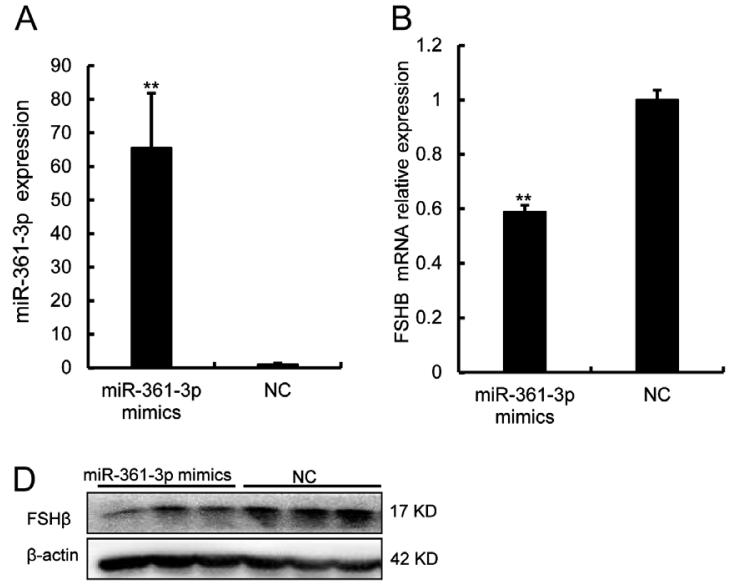

$\mathrm{E}$
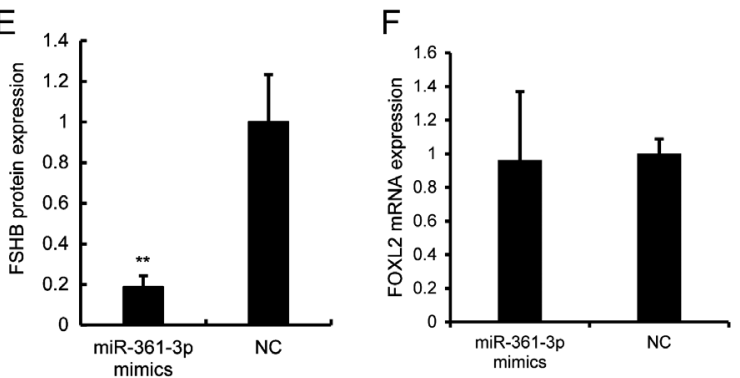

C
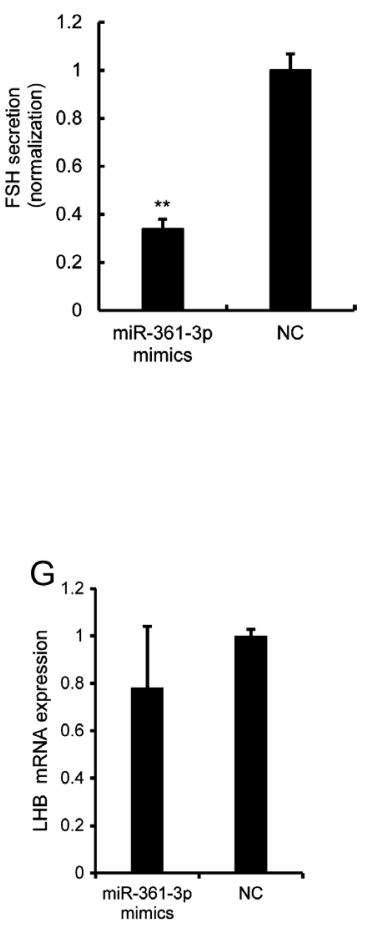

Figure 3 miR-361-3p overexpression significantly decreases FSH synthesis and secretion. (A) Examination of miR-361-3p levels following transfection of miR-361-3p mimics or a negative control (NC) into pig anterior pituitary cells. (B) Examination of $F S H B$ mRNA expression in cells transfected with miR-361-3p mimics or the NC. (C) Western blotting to examine FSHB protein expression in cells transfected with miR-

361-3p mimics or the NC. (D) Comparison of the relative expression of FSHB following densitometry analysis of the blots shown in (C), densitometry analysis was conducted by ImageJ software. (E) Quantification of FSH in the culture supernatants of cells transfected with miR-361-3p mimics or the NC.

(F) Examination of FOXL2 mRNA expression in cells transfected with miR-361-3p mimics or the NC. (G) Examination of $\angle H B$ mRNA expression in cells transfected with miR361-3p mimics or the NC. ${ }^{*} P<0.05$; $* * P<0.01$.
miR-361-3p abundance within the cell (65-fold increase, $P<0.01$; Fig. 3A). Examination of the intracellular FSHB mRNA abundance as well as FSHB protein levels also indicated that the upregulation of miR-361-3p expression significantly decreased FSHB expression, both at the mRNA ( $41 \%$ decrease, $P<0.01$, Fig. $3 \mathrm{~B})$ and protein level ( $81 \%$ decrease, Fig. $3 \mathrm{D}$ and $\mathrm{E})$. To test whether miR-361-3p overexpression also affected FSH secretion, we examined FSH levels by RIA in the culture supernatants $24 \mathrm{~h}$ after transfection, and found a significant decrease (62\% decrease, $P<0.01$, Fig. 3 C). Notably, overexpression of miR-361-3p in pituitary cells did not alter non-target genes (FOXL2 and $L H B$ ) expression (Fig. 3F and G).

\section{Confirmation of miR-361-3p function by miR-361-3p inhibition and target blockade}

As the mimic-induced overexpression of miR-361-3p led to a significant decrease in FSH synthesis and secretion, we tested whether downregulation of miR-361-3p exerted the opposite effect. Porcine anterior pituitary cells were transfected with miR-361-3p inhibitors or an $\mathrm{i}-\mathrm{NC}$. As expected, miR-361-3p inhibitor transfection significantly decreased miR-361-3p abundance in the pituitary cells $(7 \%$ of that in i-NC-transfected cells, $P<0.01$, Fig. 4A). In contrast to treatment with miR361-3p mimics (Fig. 3B, C, D and E), treatment with the miR-361-3p inhibitor significantly increased $F S H B$ mRNA abundance $(40 \%$ increase over that in the $\mathrm{i}-\mathrm{NC}$ transfected cells, $P<0.05$, Fig. 4B) and protein secretion
(60\% increase over that in the i-NC-transfected cells, Fig. 4C). Additionally, to test whether the miR-361-3p effect on FSH was specific, siRNA candidate (siRNA1) targeting $F S H B$ was designed, $F S H$ production was blocked (both at the mRNA and secreted protein level; $91 \%, 69 \%$ decrease, respectively, $P<0.01$, Fig. 4D and E) in the siRNA-transfected cells. Then, porcine anterior pituitary cells were co-transfected with siRNA-1 and the miR-361-3p inhibitor, and we found that siRNA blocked the miR-361-3p inhibitor-induced upregulation of both FSHB mRNA synthesis (Fig. 4F) and FSH secretion (Fig. 4G), while both siRNA and inhibitor have no significant effect on non-target genes (FOXL2, LHB) expression (Fig. $4 \mathrm{H}$ and $\mathrm{I}$ ), indicating that this miRNA specifically regulates $\mathrm{FSH}$ by binding $F S H B$.

\section{Discussion}

The role of miRNAs in pituitary hormone regulation is becoming increasingly evident, with recent studies indicating an involvement in gonadotropin regulation from the perspective of both miRNA biogenesis and specificity. In the present study, we found miR-361-3p could regulate $\mathrm{FSH}$ synthesis by targeting $F S H B$ in pig pituitary cells. MiR-361-3p is an X-linked miRNA, and is annotated only in certain mammals (humans, mouse, rat and the pig) thus far, with the evidence indicating that the encoding gene (miR-361) is conserved in these species. In addition to the pituitary gland, miR-361-3p is reported to be expressed at other sites, including skeletal 

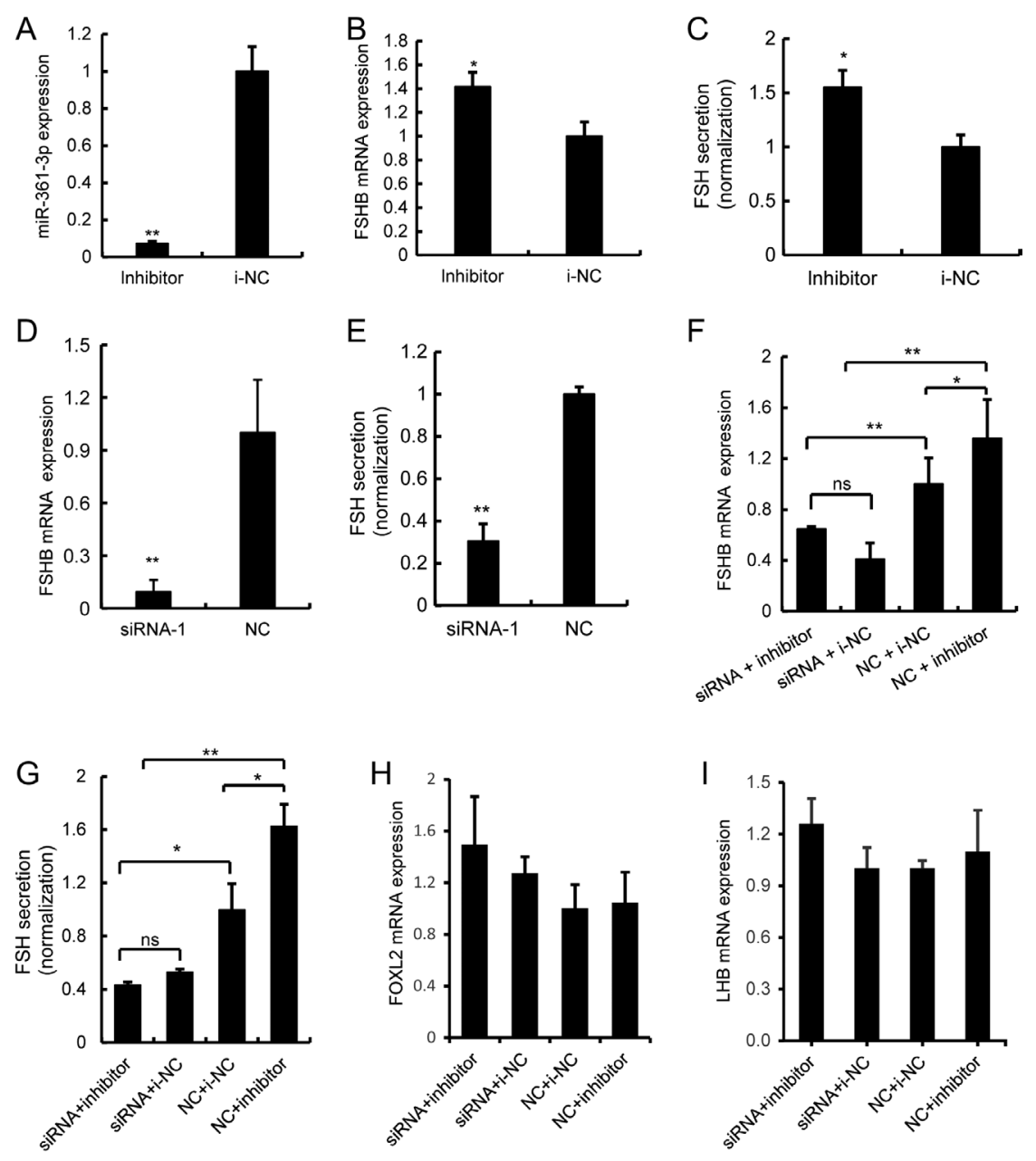

Figure 4 Confirmation of miR-361-3p function by miR-361-3p inhibition and blockade of its target. (A) Pig anterior pituitary cells were transfected with miR-361-3p inhibitors or an inhibitor negative control (i-NC), and the relative expression of miR-361-3p was examined by qRT-PCR. (B) Examination of FSHB mRNA expression in cells transfected with miR-361-3p inhibitors or the $\mathrm{i}-\mathrm{NC}$. (C) Examination of secreted FSH levels following transfection with miR-361-3p inhibitors or the i-NC. (D) Examination of FSHB mRNA expression in cells transfected with the optimal FSH-targeted siRNA (siRNA-1, 60 pmol siRNA in $2 \mathrm{~mL}$ culture media) or the i-NC. (E) Examination of secreted $\mathrm{FSH}$ secretion following transfection of cells with siRNA-1 or the i-NC.

(F) Examination of $F S H B$ mRNA expression following transfection of cells with siRNA-1 or siRNA control (NC) in combination with the miR-361-3p inhibitor or the corresponding controls. (G) Examination of $F S H B$ mRNA expression following transfection of cells with siRNA-1 in combination with the miR-361-3p inhibitor or the corresponding controls. (H) Examination of FOXL2 mRNA expression. (I) Examination of $L H B$ mRNA expression. $* P<0.05 ; * * P<0.01 ;$ ns, not significant. muscle (Nielsen et al. 2010), blood (Roth et al. 2012) and the central nervous system (Wang et al. 2011). Additionally, abnormal expression has been reported in multiple cancers, including lung cancer (Du et al. 2013), acute lymphoblastic leukemia (Schotte et al. 2011) and human oral cancer (Tanaka et al. 2011).

To date, little is known about miRNA-361-3p function. It was first identified as a potential regulator of FSH by our group, and was found to be downregulated in the primary porcine pituitary cells after $\mathrm{GnRH}$ treatment, with alterations in its abundance leading to changes in FSH secretion (Ye et al. 2013). In this study, we comprehensively examined FSH (FSHB) as well as miR-361-3p expression at different time points and different doses of GnRH treatment. We observed that miR-361-3p expression was negatively related to FSH expression in vitro, pituitary cells in response to $\mathrm{GnRH}$ and in vivo pituitary tissues. GnRH showed a dosedependent increase in FSH but decrease in miR-361-3p abundance. Interestingly, time course of GnRH treatment showed that the miR-361-3p expression was recovered at $24 \mathrm{~h}$ after $100 \mathrm{nM} \mathrm{GnRH}$, compared to $12 \mathrm{~h}$, while its target protein FSHB showed less significant decrease. The recovery of miR-361-3p in response to $\mathrm{GnRH}$ was quite similar to the observations reported in several studies (Yuen et al. 2009, Lannes et al. 2016), in which the expression of miR-132/212 and miR-125b tended to restore to steady-state level after $24 \mathrm{~h} \mathrm{GnRH}$ treatment, where miR-125b recovery was mediated by inactivating the methylation effect of NSun2 methyltransferase on miR-125b gene. Obviously, it is possible that a similar negative feedback is involved in the recovery of miR361-3p abundance in response to long-time GnRH stimulation. Nevertheless, a more detailed study on how GnRH regulate pre-miR-361 and miR-361-3p expression will be needed in the future.

In addition, bioinformatic prediction indicated that miR-361-3p may potentially target $F S H B$, as the mRNA contained a highly conserved binding site for miR361-3p in the 3'-UTR. A Dual-Luciferase Reporter Assay showed miR-361-3p significantly decrease $(P<0.01)$ FSHB 3'-UTR luciferase activity, although only $20 \%$ decrease in activity were observed, which may be related to the different response capability of different cell model. The relationship between miR-361-3p and FSHB was further confirmed by a Dual-Luciferase Reporter Assay using reporter plasmids containing either a mutation or deletion in the miR-361-3p-binding site. 
These results suggest that mutations in FSHB 3'-UTR (such as SNPs) may alter the affinity to miR-361-3p, and thus exert effects on fertility. This could possibly in a manner similar to that described by Clop et al. (Clop et al. 2006), where mutations in the myostatin 3'UTR altered binding to miR-1 and miR-206 and resulted in translational repression of the myostatin gene.

In the present study, we show that miR-361-3p overexpression significantly decreased FSHB expression at the mRNA and protein level, and reduced the levels of the secreted hormone. Conversely, miR-361-3p blockade upregulated FSH production (at the mRNA as well as secreted protein levels), and this effect was blocked by an FSH-targeted siRNA, without altering other non-target genes ( $F O X L 2, L H B)$ expression. Those results further confirmed a direct effect of miR-361-3p on FSH. Interestingly, miR-361-3p blockade using an inhibitor appeared to exert a less powerful effect than overexpression. This may be attributable to an offset effect due to endogenous miR-361-3p and isomiRNAs, or other yet unidentified miR-361-3p family members (Cloonan et al. 2011). Alternately, miR-361-3p knockdown in pituitary cells may be compensated by other pathways. As described by Wang et al., the pituitary gonadotrope expression of miR-361-3p as well as other miRNAs potentially targeting $F S H B$ was significantly suppressed in Dicer-CKO mice, but that of FSHB was also decreased (Wang et al. 2014). It is possible that $\mathrm{GnRH}$ treatment alters the expression of other miRNAs; several studies have shown that $\mathrm{GnRH}$ induces miR-132/212 expression in gonadotrope L $\beta T 2$ cells (Yuen et al. 2009). Recently, GnRH-induced FSH synthesis in both rat pituitary and mouse L $\beta$ T2 cells was shown to be dependent on miR-132/212 (Lannes et al. 2015). Supporting a role for other miRNAs in the regulation of FSH synthesis, the present study reveals that miR-361-3p plays an inhibitory role in FSH biosynthesis by targeting $F S H B$.

In addition to miR-361-3p effect on pituitary $\mathrm{FSH}$, miR-361-3p potentially involved in other reproduction processes was also detected in testis (Wu et al. 2014), ovary (Veiga-Lopez et al. 2013) and bovine follicular fluid (Sohel et al. 2013). Recent study (Sontakke et al. 2014) showed miRNA-361-3p was differentially expressed in ovarian development-related processes. Compared to large healthy follicles, miR-361-3p was significantly upregulated in large atretic follicles that involved the depletion of FSH (Ginther et al. 1999, Ilha et al. 2015). Taken together, miR-361-3p may participate in multiple fertility processes across HPG axis, although many details (such as whether miR-361-3p directly regulate ovary or testis development and how miR-361-3p regulate animal reproduction across HPG axis) remain to be elucidated in the future.

In summary, this study provides strong evidence to support that miR-361-3p directly targets FSHB and negatively regulates its expression in porcine primary anterior pituitary cells. Our findings thus identify a new posttranscriptional regulator of $\mathrm{FSH}$ synthesis. As both miR-361-3p and its binding site on FSHB mRNA are conserved among humans, mice, rats and pigs, these findings also provide a therapeutic candidate strategy for treating FSH-secreting adenomas and enrich the mechanism underling animal reproduction regulation.

\section{Declaration of interest}

The authors declare that there is no conflict of interest that could be perceived as prejudicing the impartiality of the research reported.

\section{Funding}

This work was supported by grants from the Chinese National Key Project (2016YFD0500503), the National Basic Research Program of China (973 Program, 2013CB127304 and 2011CB944200), the Natural Science Foundation of China program (31472163, 31272529), the Key Project of Guangdong Provincial Nature Science Foundation (S2013020012766) and the Key Project of Transgenic Animal (2014ZX0800948B).

\section{Author contribution statement}

$\mathrm{R} Y, M \mathrm{~L}$ and $\mathrm{C} \mathrm{L}$ performed experiment and data analysis; Q Q, T C, X C, S W, G S, L W and X Z participated in sample collection and data analysis; $R Y, C L$ and $Y Z$ participated in drafting the manuscript; and Q J, Q X and $Y Z$ conceived of the study, participated in its design and coordination, and helped to draft the manuscript.

\section{References}

Ambros V 2004 The functions of animal microRNAs. Nature 431 350-355. (doi:10.1038/nature02871)

Barb C, Barrett J, Wright J, Kraeling R \& Rampacek G 1990 Opioid modulation of LH secretion by pig pituitary cells in vitro. Journal of Reproduction and Fertility 90 213-219. (doi:10.1530/jrf.0.0900213)

Bernard DJ, Fortin J, Wang Y \& Lamba P 2010 Mechanisms of FSH synthesis: what we know, what we don't, and why you should care. Fertility and Sterility 93 2465-2485. (doi:10.1016/j.fertnstert.2010.03.034)

Chomczynski P 1993 A reagent for the single-step simultaneous isolation of RNA, DNA and proteins from cell and tissue samples. Biotechniques 15 532-534, 536-537.

Cloonan N, Wani S, Xu Q, Gu J, Lea K, Heater S, Barbacioru C, Steptoe AL, Martin HC \& Nourbakhsh E 2011 MicroRNAs and their isomiRs function cooperatively to target common biological pathways. Genome Biology 12 R126. (doi:10.1186/gb-2011-12-12-r126)

Clop A, Marcq F, Takeda H, Pirottin D, Tordoir X, Bibé B, Bouix J, Caiment F, Elsen J-M \& Eychenne F 2006 A mutation creating a potential illegitimate microRNA target site in the myostatin gene affects muscularity in sheep. Nature Genetics 38 813-818. (doi:10.1038/ng1810)

Dang Y, Zhao S, Qin Y, Han T, Li W \& Chen Z-J 2015 MicroRNA-22-3p is down-regulated in the plasma of Han Chinese patients with premature ovarian failure. Fertility and Sterility 103 802-807, e801. (doi:10.1016/j. fertnstert.2014.12.106)

Du L, Borkowski R, Zhao Z, Ma X, Yu X, Xie X-J \& Pertsemlidis A 2013 A high-throughput screen identifies miRNA inhibitors regulating 
lung cancer cell survival and response to paclitaxel. RNA Biology $\mathbf{1 0}$ 1700-1713. (doi:10.4161/rna.26541)

Ginther O, Bergfelt D, Kulick L \& Kot K 1999 Selection of the dominant follicle in cattle: establishment of follicle deviation in less than 8 hours through depression of FSH concentrations. Theriogenology 52 1079-1093. (doi:10.1016/S0093-691X(99)00196-X)

Hasuwa H, Ueda J, Ikawa M \& Okabe M 2013 miR-200b and miR-429 function in mouse ovulation and are essential for female fertility. Science 341 71-73. (doi:10.1126/science.1237999)

Ilha GF, Rovani MT, Gasperin BG, Antoniazzi AQ, Gonçalves PBD, Bordignon V \& Duggavathi R 2015 Lack of FSH support enhances LIF-STAT3 signaling in granulosa cells of atretic follicles in cattle. Reproduction 150 395-403. (doi:10.1530/REP-15-0026)

Karginov FV, Cheloufi S, Chong MM, Stark A, Smith AD \& Hannon GJ 2010 Diverse endonucleolytic cleavage sites in the mammalian transcriptome depend upon microRNAs, Drosha, and additional nucleases. Molecular cell 38 781-788. (doi:10.1016/j. molcel.2010.06.001)

Kiezun M, Smolinska N, Maleszka A, Dobrzyn K, Szeszko K \& Kaminski T 2014 Adiponectin expression in the porcine pituitary during the estrous cycle and its effect on LH and FSH secretion. American lournal of Physiology-Endocrinology and Metabolism 307 E1038-E1046. (doi:10.1152/ajpendo.00299.2014)

Lannes J, L'hôte D, Garrel G, Laverrière J-N, Cohen-Tannoudji J \& Quérat B 2015 A microRNA-132/212 pathway mediates GnRH activation of FSH expression. Molecular Endocrinology 29 364-372. (doi:10.1210/ me.2014-1390)

Lannes J, L'hôte D, Fernandez-Vega A, Garrel G, Laverrière J-N, JoëlleCohen-Tannoudji J-C-T \& Quérat B 2016 A regulatory loop between miR-132 and miR-125b involved in gonadotrope cells desensitization to GnRH. Scientific Reports 6 31563. (doi:10.1038/srep31563)

Lin J, Barb C, Kraeling R \& Rampacek G 2003 Growth hormone releasing factor decreases long form leptin receptor expression in porcine anterior pituitary cells. Domestic Animal Endocrinology 24 95-101. (doi:10.1016/S0739-7240(02)00209-6)

Nemoto T, Mano A \& Shibasaki T 2012 Increased expression of miR-325$3 p$ by urocortin 2 and its involvement in stress-induced suppression of LH secretion in rat pituitary. American Journal of PhysiologyEndocrinology and Metabolism 302 E781-E787. (doi:10.1152/ ajpendo.00616.2011)

Nielsen M, Hansen J, Hedegaard J, Nielsen R, Panitz F, Bendixen C \& Thomsen B 2010 MicroRNA identity and abundance in porcine skeletal muscles determined by deep sequencing. Animal Genetics 41 159-168. (doi:10.1111/j.1365-2052.2009.01981.x)

Okada Y, Murota-Kawano A, Kakar SS \& Winters SJ 2003 Evidence that gonadotropin-releasing hormone $(\mathrm{GnRH})$ II stimulates luteinizing hormone and follicle-stimulating hormone secretion from monkey pituitary cultures by activating the $\mathrm{GnRH}$ I receptor. Biology of Reproduction 69 1356-1361. (doi:10.1095/biolreprod.103.016162)

Repetto E, Briata P, Kuziner N, Harfe BD, McManus MT, Gherzi R, Rosenfeld MG \& Trabucchi M 2012 Let-7b/c enhance the stability of a tissue-specific mRNA during mammalian organogenesis as part of a feedback loop involving KSRP. PLoS Genetics 8 e1002823. (doi:10.1371/ journal.pgen.1002823)

Roth C, Stückrath I, Pantel K, Izbicki JR, Tachezy M \& Schwarzenbach H 2012 Low levels of cell-free circulating miR-361-3p and miR-625* as blood-based markers for discriminating malignant from benign lung tumors. PLOS ONE 7 e38248. (doi:10.1371/journal.pone.0038248)

Schotte D, Moqadam FA, Lange E-Turenhout E, Chen C, Van IJcken W, Pieters R \& den Boer M 2011 Discovery of new microRNAs by small RNAome deep sequencing in childhood acute lymphoblastic leukemia. Leukemia 25 1389-1399. (doi:10.1038/leu.2011.105)
Sohel MMH, Hoelker M, Noferesti SS, Salilew-Wondim D, Tholen E, Looft C, Rings F, Uddin MJ, Spencer TE, Schellander K et al. 2013 Exosomal and non-exosomal transport of extra-cellular microRNAs in follicular fluid: implications for bovine oocyte developmental competence. PLOS ONE 8 e78505. (doi: 10.1371/journal.pone.0078505)

Sontakke SD, Mohammed BT, McNeilly AS \& Donadeu FX 2014 Characterization of microRNAs differentially expressed during bovine follicle development. Reproduction 148 271-283. (doi:10.1530/REP14-0140)

Tanaka H, Nakashiro K-i, Oka R, Nishikawa Y, Goda H \& Hamakawa H 2011 MicroRNA-361-3p functions as an oncogenic microRNA in human oral cancer cells. Cancer Research 71 145-145. (doi:10.1158/15387445.AM2011-145)

Thackray VG, Mellon PL \& Coss D 2010 Hormones in synergy: regulation of the pituitary gonadotropin genes. Molecular and Cellular Endocrinology 314 192-203. (doi:10.1016/j.mce.2009.09.003)

Veiga-Lopez A, Luense LJ, Christenson LK \& Padmanabhan V 2013 Developmental programming: gestational bisphenol-A treatment alters trajectory of fetal ovarian gene expression. Endocrinology 154 1873-1884. (doi:10.1210/en.2012-2129)

Wang W-X, Huang Q, Hu Y, Stromberg AJ \& Nelson PT 2011 Patterns of microRNA expression in normal and early Alzheimer's disease human temporal cortex: white matter versus gray matter. Acta Neuropathologica 121 193-205. (doi:10.1007/s00401-010-0756-0)

Wang H, Graham I, Hastings R, Gunewardena S, Brinkmeier ML, Conn PM, Camper SA \& Kumar TR 2014 Gonadotrope-specific deletion of Dicer results in severely suppressed gonadotropins and fertility defects. Journal of Biological Chemistry 290 2699-2714. (doi:10.1074/ jbc.M114.621565)

Wu J, Zhu H, Song W, Li M, Liu C, Li N, Tang F, Mu H, Liao M \& Li X 2014 Identification of conservative microRNAs in Saanen dairy goat testis through deep sequencing. Reproduction in Domestic Animals 49 32-40. (doi:10.1111/rda.12217)

Wurmbach E, Yuen T, Ebersole BJ \& Sealfon SC 2001 Gonadotropinreleasing hormone receptor-coupled gene network organization. Journal of Biological Chemistry 276 47195-47201. (doi:10.1074/jbc. M108716200)

Ye R-S, Xi Q-Y, Qi Q, Cheng X, Chen T, Li H, Kallon S, Shu G, Wang S-B \& Jiang Q-Y 2013 Differentially expressed miRNAs after GnRH treatment and their potential roles in FSH regulation in porcine anterior pituitary cell. PLOS ONE 8 e57156. (doi:10.1371/journal.pone.0057156)

Yuen T, Ruf F, Chu T \& Sealfon SC 2009 Microtranscriptome regulation by gonadotropin-releasing hormone. Molecular and Cellular Endocrinology 302 12-17. (doi:10.1016/j.mce.2008.12.013)

Zhang Z, Florez S, Gutierrez-Hartmann A, Martin JF \& Amendt BA 2010 MicroRNAs regulate pituitary development, and microRNA 26b specifically targets lymphoid enhancer factor 1 (Lef-1), which modulates pituitary transcription factor 1 (Pit-1) expression. Journal of Biological Chemistry 285 34718-34728. (doi:10.1074/jbc.M110.126441)

Zhang N, Lin J-k, Chen J, Liu X-f, Liu J-l, Luo H-s, Li Y-q \& Cui S 2013 MicroRNA 375 mediates the signaling pathway of corticotropin-releasing factor (CRF) regulating pro-opiomelanocortin (POMC) expression by targeting mitogen-activated protein kinase 8. Journal of Biological Chemistry 288 10361-10373. (doi:10.1074/jbc.M112.425504)

Received 11 July 2016

First decision 29 July 2016

Revised manuscript received 30 November 2016

Accepted 19 December 2016 